\title{
Optimization Audicor for Normal and Abnormal Heart Sounds Characteristic
}

\author{
Dedi Kurniadi ${ }^{\text {a,1,*}, ~ S u r y a ~ Y o n d r i ~}{ }^{\text {a }}$, Albar ${ }^{\text {a }}$, Roza Susanti ${ }^{\text {a }}$, David Eka Putra ${ }^{\text {a }}$, Gwo-Jia Jong ${ }^{\text {b }}$ \\ ${ }^{a}$ Department of Electrical Engineering, Politeknik Negeri Padang, Padang, Indonesia \\ ${ }^{\mathrm{b}}$ Department Electronic Department, National Kaohsiung University of Science and Technology, Taiwan \\ 1dikurniadi@pnp.ac.id \\ * corresponding author
}

\section{ARTICLE INFO}

Article history

Received May 13, 2020

Revised June 8, 2020

Accepted June 10, 2020

Keywords

signal processing

fast fourier transform

principal component analysis

phonocardiograph

heart sound

\section{ABSTRACT}

Heart Sounds are important things in the human body that can deliver information related to the heart condition. However, a recorded signal such as PCG and ECG that getting through Audicor still contain unexpected components or noise while the recording process happens it makes the result data from Audicor cannot directly use to recognize the condition of the heart. This research presents signal processing and data analysis to suppress the noise of the heart sounds that getting while the process of recording data happens. The cleaned heart sound will be processed in feature extraction by using FFT and PCA that capable to produce the feature both of the normal and abnormal heart sounds. For the normal case, we get the data from some healthy volunteers recorded by using Audicor. While the abnormal heart sound we focus to observe the data that contain Ventricular Septal Defect (VSD) that getting from a partner hospital. As a result, feature both normal and abnormal heart sounds can be separated.

This is an open access article under the CC-BY-SA license.

\section{Introduction}

In heart auscultation, heart sounds are the most significant cue to recognize heart condition [1]. The function of the heart is pumping the blood throughout the body and receive it again after getting the cleaning process in the lungs. More specifically the dirty blood with poor oxygen from the body that passes through the vein vessel will be transferred from the right side of the heart into the right atrium and will be transferred into the right ventricle through the tricuspid valve. Then the blood from the right ventricle will be pumped into the lung through the pulmonary valve. After that, the blood flows through the small size of the vascular vessel surrounding the air pockets in the lungs to absorb oxygen and release carbon dioxide, and then blood will flow back to the heart. On the left side of the heart, the oxygen-rich blood will transfer into the left atrium through the mitral valve, then the blood will be going to the throughout the body except lungs through aortic valve [7][8][9]. In the pumping process of the blood throughout the body, the heart will produce the sound continuously that commonly known as lub and dub. Sounds that produced by the heart is coming from the closing of the heart valves. When the tricuspid and mitral valve is closing that will produce lub sound known as first heart sounds or S1. Similarly to the dub sound or second heart sound (S2), it exists because both the aortic and pulmonic valves are closing [10]. 
Beside S1 and S2 also have other additional heart sounds that consist of the third heart sound or S3, fourth heart sound or S4, and murmurs. These three additional heart sounds indicate the abnormal heart sounds [11].

Heart sounds that are getting through the sounds that produce when the circulation process capable to give the information related to the condition of the patient heart [2]. In general, the heart can produce two main components in every cycle that consist of the first heart sound and second heart that is commonly known as S1 and S2. Due to the abnormal condition or because of the age, the heart will also produce an extra component of the sound that consists of third and fourth heart sound or commonly known as S3 and S4, where these extra heart sounds have a lower amplitude compared to the main component of heart sound (S1 and S2) of the heart sounds [3][4]. Beside S3 and S4 also there are systolic and diastolic murmurs. These heart sounds are recording through a portable Audicor that placing in some positions in the chase of the patient such as mitral, tricuspid, aortic, and Pulmonic. By using some positions of the chase of the patient it can get the time duration and frequency value of the heart sound in one cycle. Regarding these parameters, researchers applied their algorithms for doing some observation and decide on the condition of the heart sound signals that they are observed. Observation of the normal and abnormal heart sounds can be done through the time domain and frequency domain. P.S Vikh et al have been conducted and focused their research for detecting the normal and abnormal heart sounds by using Short Time Fourier Transform (STFT) and Continuous Wavelet Transform (CWT) [5]. A. Gharehbaghi et al created a methodology to classify heart murmurs [1]. S. Barma et al implement third heart sound (S3) detection based on non-linear signal decomposition and time-frequency localization [6]. Andrizal et al use FFT to detect the data pattern categories of the combustion engine based on exhaust emission [7].

This paper implements FFT and PCA for feature extraction on normal and abnormal heart sounds. For the normal heart sounds are getting through some volunteer that has a normal heart then those recorded signal was discussing with a physician to make sure the signal possible to use as a normal signal or vise versa. Then for the abnormal heart sounds was used HS that indicate as a Ventricular Septal Defect (VSD). VSD is one of the congenital heart disease [15] that existing a hole in the septum of the muscle wall that possible to separate between right and left chambers [16] [17] [18]. In normal condition, Rich-oxygen blood will be pumped to the aorta then transfer it to the whole body from aorta and lungs. Because of the VSD safer, the blood is not directly transferred to whole the body but some blood gets push through-hole of VSD into the right ventricle. In the feature extraction approach, firstly will be done through the preprocessing technique for the recorded heart sounds that getting from Audicor. Preprocessing technique, in this case, is using IIR high pass filter in heart sounds, and for facing the ECG signal that getting from Audicor is solved through baseline removal technique, this issue is doing by using this method because the recorded data from Audicor is not always stable for the peak value of the signal so it can difficult to implement in peak detection approach. Where this peak detection is used to obtain the signal reference that needs in the segmentation process. The aim of the segmentation process is for dividing the signal into some segments (every cycle) based on peak detection in ECG signal. After that, the feature extraction technique is implemented to get the parameter both of the normal and abnormal heart sounds.

\section{Material and Method}

\subsection{Heart Sounds Auscultation Process}

In the Auscultation process, the research purposed to implement heart sounds analysis according to heart anatomy. In normal conditions, physicians normally can measure and observe the condition of the patient hearts through a traditional way which is placing the stethoscope on the chest patient. In order to measure the sound of the patient heart, four common places normally used for 
auscultation process, which are mitral, tricuspid, pulmonic, and aortic as shown in the following figure [12].

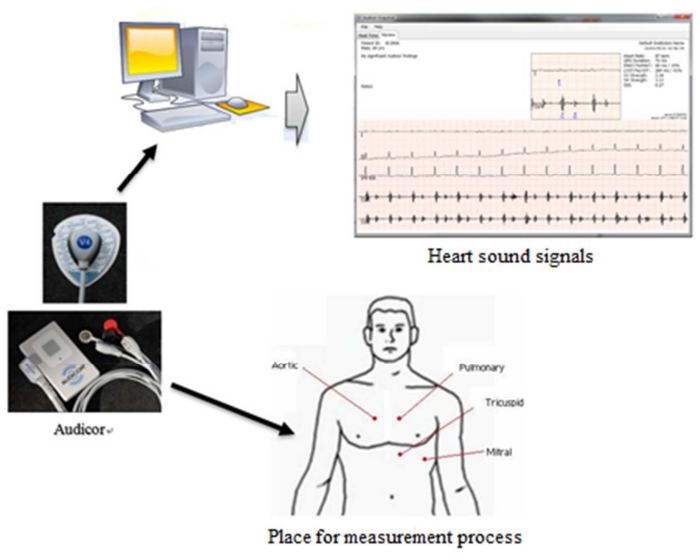

Fig 1. Measurement process of the heart sounds

Phonocardigraph is one of the most common methods implemented for heart sounds auscultation. In recording the heart sounds, this paper applied the Audicor for getting the heart sounds and Electrocardiograph (ECG) signals. The recorded signals are going to be processed and analyzed to obtain the characteristic both of normal and abnormal heart sounds. These characteristics will be discussed with an expert or physician in heart disease. Then, the recorded ECG signals are used as a parameter in the signal processing approach [13]. For supporting data, this research uses data samples both of the normal and abnormal. The data as a normal patient is obtaining through some volunteers that have a good condition in health. While the sample data that used as an abnormal patient are getting from a cooperative hospital. So, the normal and abnormal heart sound we have 20 sample data respectively.

This paper has some steps in the signal processing approach that can be used for separating the feature each of the normal and abnormal heart sounds that consist as follows:

\subsection{Flowchart of The Signal Processing Approach}

There are three steps in the signal processing approach that consists of preprocessing, segmentation, and feature extraction as shown in figure 3.

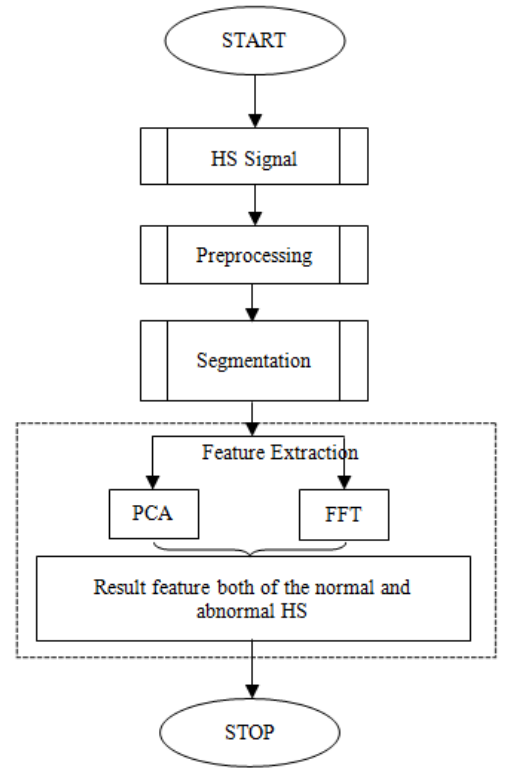

Fig 2. Flowchart of the process 
The flowchart illustrates the process of the signal processing approach that is used in this research.

- Firstly the data are recorded through an Audicor that will produce both of the PCG and ECG signals.

- Raw data from Audicor are going to process through a preprocessing technique. In this stage, data will be cleaned from the noise to get the real data of PCG and ECG. In this case, the bandpass filter is implemented (BPF) for the PCG signal to suppress the noise that has been got when the recording data is happening. However, for ECG signal is used Baseline Removal or Baseline Wandering technique for facing the noise before implementing the peak detection.

- In the stage of segmentation, this research [14] applied wavelet decomposition and reconstruction to get the detail and approximation of the frequency difference of the original PCG signal, that is capable of separating PCG signals into several parts according to peak detection and implementation of cross-correlation and normalization cross-correlation to choose the best signal that can be processed in the next step.

- Feature extraction is the most important stage in this research because this stage will produce the result that makes us capable to recognize and distinguish between normal and abnormal heart sounds. This step implements the FFT and PCA.

\subsection{Segmentation Process}

Segmentation is the process that implements cross-correlation and normalizes cross-correlation for separating the HS signal into some parts. By using peak detection as a reference signal for the cutting process of HS signal, the research capable to choose a better signal from some segments that have been made previously. This selected signal is getting through cross-correlation as shown in equation 1 dan normalize cross-correlation in equation 2.

$$
\operatorname{Corr} x, y=\sum_{N=0}^{N-1} x[n] y[n]
$$

Equation 1 illustrates the working principle of cross-correlation, where the signal $\mathrm{x}$ will be compared with signal $\mathrm{y}$ and $\mathrm{x}$ as a reference signal. The output number will get an indication as a similarity level, the high number considered as a high similarity. After that, we create standardize for cross-correlation known as normalized cross-correlation.

\section{Results and Discussion}

As mentioned earlier, this literature review will be limited to journals published in 2010 through 2018. After facing some steps in signal processing approach such preprocessing for PCG and ECG signals so it is continued with the segmentation process for heart sound signals. Where the heart sound signals will be classified into some parts based on peak detection that implements crosscorrelation and normalizes cross-correlation results to get the best signal that is going to be processed in the next steps.

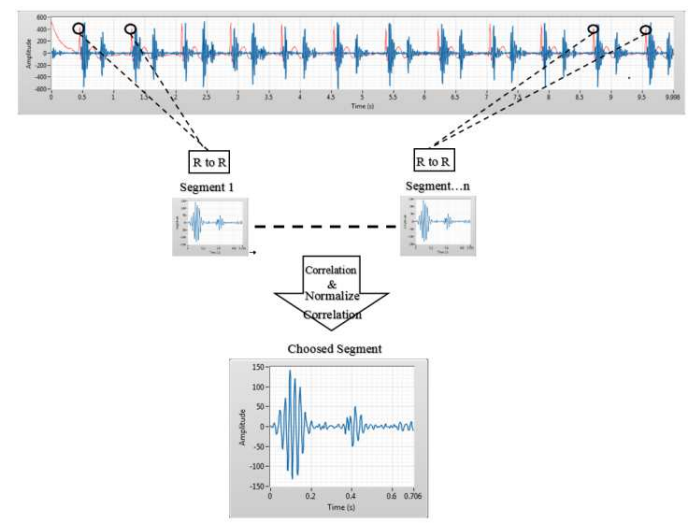

Fig 3. Segmentation Result 
In feature extraction, this research use FFT to observe both of the normal and abnormal heart sounds based on the amplitude value of the heart sounds signal and this result will be used as an input in a statistical data observation on PCA. PCA is used to do the observation and analyze the different parameters both of normal and abnormal heart sounds.

Table 1. Summary Statistics (Quantitative data) for Normal HS

\begin{tabular}{cccccccc}
\hline Variable & Obs & miss data & $\begin{array}{c}\text { without } \\
\text { miss data }\end{array}$ & $\begin{array}{c}\text { Minimu } \\
\mathrm{m}\end{array}$ & Max & Mean & Std. deviation \\
\hline P1 & 45 & 0 & 45 & 0.693 & 14.970 & 5.335 & 3.518 \\
P2 & 45 & 0 & 45 & 0.416 & 15.003 & 4.586 & 3.569 \\
P3 & 45 & 0 & 45 & 0.814 & 19.422 & 7.102 & 5.316 \\
P4 & 45 & 0 & 45 & 0.538 & 11.578 & 3.906 & 2.959 \\
P5 & 45 & 0 & 45 & 0.546 & 6.630 & 2.508 & 1.411 \\
P6 & 45 & 0 & 45 & 0.190 & 6.610 & 2.310 & 1.680 \\
P7 & 45 & 0 & 45 & 0.156 & 3.310 & 1.438 & 0.826 \\
P8 & 45 & 0 & 45 & 0.208 & 5.052 & 1.627 & 1.203 \\
P9 & 45 & 0 & 45 & 0.129 & 6.926 & 1.863 & 1.549 \\
P10 & 45 & 0 & 45 & 0.323 & 13.002 & 2.948 & 2.933 \\
P11 & 45 & 0 & 45 & 0.095 & 6.057 & 2.527 & 1.773 \\
P12 & 45 & 0 & 45 & 0.174 & 4.893 & 1.642 & 1.153 \\
P13 & 45 & 0 & 45 & 0.221 & 8.693 & 3.192 & 2.407 \\
P14 & 45 & 0 & 45 & 0.332 & 5.868 & 1.934 & 1.516 \\
P15 & 45 & 0 & 45 & 0.289 & 5.086 & 1.547 & 1.022 \\
P16 & 45 & 0 & 45 & 0.252 & 7.379 & 2.537 & 1.690 \\
P17 & 45 & 0 & 45 & 0.251 & 6.427 & 1.660 & 1.247 \\
P18 & 45 & 0 & 45 & 0.178 & 10.904 & 3.373 & 2.635 \\
P19 & 45 & 0 & 45 & 0.377 & 14.714 & 5.392 & 3.839 \\
P20 & 45 & 0 & 45 & 0.235 & 14.714 & 5.251 & 3.970 \\
\hline & & & & & & & \\
\hline
\end{tabular}

According to both of the normal and abnormal heart sound data that consist of 20 sample in normal condition and 20 sample data for the abnormal condition too, we can see the difference between normal and abnormal heart sound through the maximum value of summary statistic table. This condition can be more clear by using the graph to separate both of the normal and abnormal HS. 
Table 1. Summary Statistics (Quantitative data) for Abnormal HS

\begin{tabular}{|c|c|c|c|c|c|c|c|}
\hline Variable & Obs & $\begin{array}{l}\text { miss } \\
\text { data }\end{array}$ & $\begin{array}{l}\text { without } \\
\text { miss data }\end{array}$ & Minimum & Max & Mean & Std. deviation \\
\hline $\mathrm{P} 21$ & 45 & 0 & 45 & 0.302 & 64.381 & 19.610 & 19.568 \\
\hline $\mathrm{P} 22$ & 45 & 0 & 45 & 0.210 & 15.123 & 4.553 & 3.817 \\
\hline $\mathrm{P} 23$ & 45 & 0 & 45 & 0.400 & 32.091 & 10.374 & 8.827 \\
\hline $\mathrm{P} 24$ & 45 & 0 & 45 & 0.324 & 19.769 & 8.127 & 6.246 \\
\hline $\mathrm{P} 25$ & 45 & 0 & 45 & 0.195 & 41.945 & 16.285 & 14.106 \\
\hline $\mathrm{P} 26$ & 45 & 0 & 45 & 0.292 & 45.054 & 12.783 & 14.219 \\
\hline P27 & 45 & 0 & 45 & 0.247 & 10.148 & 3.804 & 2.620 \\
\hline $\mathrm{P} 28$ & 45 & 0 & 45 & 0.357 & 12.813 & 5.136 & 3.613 \\
\hline P29 & 45 & 0 & 45 & 0.116 & 78.672 & 18.517 & 20.606 \\
\hline P30 & 45 & 0 & 45 & 0.313 & 16.367 & 6.221 & 4.400 \\
\hline P31 & 45 & 0 & 45 & 0.983 & 17.349 & 6.031 & 4.146 \\
\hline P32 & 45 & 0 & 45 & 0.625 & 44.381 & 12.304 & 10.921 \\
\hline P33 & 45 & 0 & 45 & 0.432 & 19.992 & 6.537 & 5.377 \\
\hline P34 & 45 & 0 & 45 & 0.336 & 38.783 & 11.317 & 11.413 \\
\hline P35 & 45 & 0 & 45 & 0.873 & 72.897 & 24.085 & 23.543 \\
\hline P36 & 45 & 0 & 45 & 0.119 & 47.955 & 12.990 & 14.219 \\
\hline P37 & 45 & 0 & 45 & 0.445 & 19.128 & 7.859 & 6.439 \\
\hline P38 & 45 & 0 & 45 & 0.108 & 40.093 & 11.397 & 12.444 \\
\hline P39 & 45 & 0 & 45 & 0.176 & 36.327 & 15.947 & 9.278 \\
\hline $\mathrm{P} 40$ & 45 & 0 & 45 & 3.267 & 59.105 & 26.926 & 16.118 \\
\hline
\end{tabular}

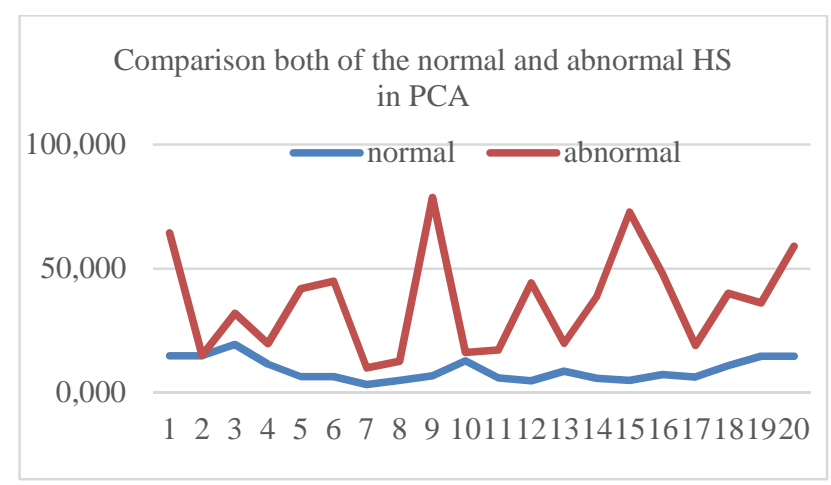

Fig 4. Graph of normal and abnormal heart sounds 
Figure 5 shows the differences between normal and abnormal heart sounds. The normal heart sounds indicated by the blue color of the line and the abnormal one shows in the red line. From the tren that showing in this graph can give the information and separate both of the normal and abnormal heart sounds

\section{Conclusion}

This research implements some steps in signal processing that consists of preprocessing, segmentation, and feature extraction. In preprocessing and segmentation technique the signal will be processed to get the real signal and ready to be used as an input for the feature extraction stage. Feature extraction implements FFT and PCA to recognize characteristics both of normal and abnormal heart sounds. For the implementation process, this paper use FFT to detect the differences between normal and abnormal heart sound based on the amplitude value, and this result also will be used as an input for statistical data processing in PCA. While for the PCA itself uses maximum value on the summary statistic table of the normal and abnormal heart sounds. These two kinds of normal and abnormal data in summary statistic data value successfully show a difference parameter between normal and abnormal heart sounds.

\section{Acknowledgment}

This research is conducted through DIPA Politeknik Negeri Padang with contract number 149/PL9.1.4/PT.01.02/2019, because of that, the author would like to say thanks to Politeknik Negeri Padang that have been funded this research.

\section{References}

[1] A. Gharehbaghi, M. Borga, B. J. Sjöberg, and P. Ask, "A novel method for discrimination between innocent and pathological heart murmurs," Med. Eng. Phys., vol. 37, no. 7, pp. 674-682, 2015.

[2] Kurniadi, D., Kung, Y.-F., Chen, Z.-H., Li, Y.-P., Hendrick, \& Jong, G.-J. (2018). "Implemented the expert system of heart disease by using SVM", 2018 IEEE International Conference on Applied System Invention (ICASI).

[3] L. Hamza Cherif, S. M. Debbal, and F. Bereksi-Reguig, "Segmentation Of Heart Sounds and Heart Murmurs," Journal of Mechanics in Medicine and Biology. 2008.

[4] Kurniadi, D., Kung, Y.-F., Chen, Z.-H., Li, Y.-P., Hendrick, \& Jong, G.-J. (2018). "Implemented the expert system of heart disease by using SVM". 2018 IEEE International Conference on Applied System Invention (ICASI).

[5] P. S. Vikhe, N. S. Nehe, and V. R. Thool, "Heart Sound Abnormality Detection Using Short Time Fourier Transform and Continuous Wavelet Transform," 2009 Second Int. Conf. Emerg. Trends Eng. Technol., no. 1, pp. 50-54, 2009.

[6] W. et al. Barma, Shovan; Chen, Bo-Wei; Ji, "Detection of the Third Heart Sound Based on Nonlinear Signal Decomposition and Time-Frequency Localization IF3.57 Q1,” vol. 63, no. 8, pp. 1718-1727, 2016.

[7] Andrizal, R. Chadry, and A. I. Suryani, "Embedded System Using Field Programmable Gate Array (FPGA) myRIO and LabVIEW Programming to Obtain Data Patern Emission of Car Engine Combustion Categories," JOIV Int. J. Informatics Vis., vol. 2, no. 2, p. 56, 2018.

[8] "How the Heart Works - Topic Overview." [Online]. Available: https://myhealth.alberta.ca/health/pages/conditions.aspx?Hwid=tx4097abc. [Accessed: 09-Apr-2018].

[9] “(18) Video Pembelajaran: Sistem Peredaran Darah Manusia - YouTube.” [Online]. Available: https://www.youtube.com/watch?v=j0t8Lif8NZc. [Accessed: 10-May-2019].

[10] A. K. Abbas and R. Bassam, "Phonocardiography Signal Processing," Synth. Lect. Biomed. Eng., vol. 4, no. 1, pp. 1-194, 2009.

[11] "Flow through the heart (video) | Khan Academy." [Online]. Available: https://www.khanacademy.org/science/health-and-medicine/circulatory-system/circulatory-systemintroduction/v/lub-dub. [Accessed: 24-Apr-2018]. 
[12] A. Mondal, P. Bhattacharya, and G. Saha, "An automated tool for localization of heart sound components S1, S2, S3 and S4 in pulmonary sounds using Hilbert transform and Heron's formula," Springerplus, vol. 2, no. 1, pp. 1$14,2013$.

[13] Aripriharta, "The Biomedical Signal Processing Combined with Internet of Things The Biomedical Signal Processing Combined with Internet of Things," 2017.

[14] M. Nassralla, Z. El Zein, and H. Hajj, "Classification of Normal and Abnormal Heart Sounds,” 2017.

[15] L. Huiying, L. Sakari, and H. Iiro, "A heart sound segmentation algorithm using wavelet decomposition and reconstruction," Proc. 19th Annu. Int. Conf. IEEE Eng. Med. Biol. Soc. 'Magnificent Milestones Emerg. Oppor. Med. Eng. (Cat. No.97CH36136), vol. 4, no. C, pp. 1630-1633, 1997.

[16] A.Wang andT. M.Bashore, Valvular Heart Disease. 2009.

[17] "Ventricular Septal Defect (VSD)." [Online]. Available: http://www.stanfordchildrens.org/ en/topic/default?id=ventricular-septal-defect-vsd-90-P01829. [Accessed: 24-Apr-2018].

[18] “Ventricular Septal Defect (VSD) in Children | Phoenix Children's Hospital Heart Center." [Online]. Available: http://heart.phoenixchildrens.org/heart-conditions/ventricular-septal-defect-vsd-children. [Accessed: 24-Apr2018].

[19] "Ventricular septal defect (video) | Khan Academy." [Online]. Available: https://www.khanacademy.org/ science/health-and-medicine/circulatory-system-diseases/acyanotic-heart-diseases/v/rn-ventricular-septal-defect. [Accessed: 24-Apr-2018] 